\title{
Ferromagnetic Layered Composites. Transfer Matrix Approach
}

\author{
H. Puszkarski ${ }^{a, *}$, M. KrawczyK ${ }^{a}$, J.-C.S. Lévy ${ }^{b}$ \\ AND D. MERCIER ${ }^{b}$ \\ ${ }^{a}$ Surface Physics Division, Faculty of Physics, Adam Mickiewicz University \\ Umultowska 85, 61-614 Poznań, Poland \\ ${ }^{b}$ Laboratoire de Physique Théorique de la Matière Condensée, case 7020 \\ Université Paris 7, 75251 Paris Cedex, France
}

(Received August 9, 2001)

\begin{abstract}
A theoretical analysis of spin-wave excitations in a ferromagnetic layered composite (ABAB...ABA; $\mathrm{A}$ and $\mathrm{B}$ are different homogeneous ferromagnetic materials) is performed by means of the transfer matrix approach. We focus our attention on two features not yet well studied in the present literature, namely, the existence of forbidden energy gaps in the spin-wave mode spectrum, as well as the properties of multilayer spin-wave mode profiles. These features are discussed in relation to several multilayer characteristics, such as the filling fraction, and the exchange or magnetization contrast; surface pinning conditions and dipolar interactions are also taken into account. The existence of forbidden gaps as well as properties of mode profiles are successfully explained by having recourse to an approximate model of independent constituent sublayers. Finally, as a byproduct of our investigation, we ascertain that the conventional test for computational convergence within the finite difference method demands to be completed by symmetry mode profile verification, in addition to standard verification of the frequency convergence alone.
\end{abstract}

PACS numbers: 75.70.-i, 75.30.Ds, 02.60.Dc, 02.70.Bf

\section{Introduction}

The interest in periodic composite materials, such as photonic [1-4], phononic [5-9], or magnonic [10-12] crystals, has been growing systematically over the last few years. Besides two- and three-dimensional periodic composites, multilayer sys-

* corresponding author; e-mail: henpusz@amu.edu.pl 
tems (providing an example of the simplest one-dimensional composite) are studied intensively, in particular, many surveys deal with magnetic multilayers [13-19]. The two reasons explain the special theoretical interest in magnetic layered composites. Local magnetic anisotropy, short-ranged exchange, and long-ranged dipolar forces are competing to define the static and dynamic properties of such materials. As a result, the magnetic arrangement of composite materials is generally complex. In the special case with an external saturating field, this arrangement becomes trivial, all spins being parallel, but the dynamic properties remain not obvious. This is the case considered here, in order to study the linear propagation of magnons through a layered composite material and to focus on dynamic properties, such as the resonance frequencies and the magnon profiles, which determine the resonance intensities in the case of a weak excitation. A complete discrete treatment of a layered system is developed in this paper, in order to obtain in a clear way the magnon profiles within a layered composite.

\section{The discretized Landau-Lifshitz equation approach}

The applied saturating field makes the direction of the static magnetization $\boldsymbol{M}_{0, l}$ parallel everywhere (because of the weakness of magnetic excitations) and is perpendicular to the dynamic component $m_{l}$ of the magnetization, which depends on the layer number $l$, with the obvious relation for the total magnetization

$$
M_{l}=M_{0, l}+m_{l} .
$$

The Landau-Lifshitz equation reads

$$
\frac{\partial \boldsymbol{M}_{l}}{\partial t}=\gamma \mu_{0} \boldsymbol{M}_{l} \times \boldsymbol{H}_{\mathrm{eff}} .
$$

Taking a resonant solution with $m_{l} \approx \exp (-\mathrm{i} \omega t)$ one obtains

$$
-\mathrm{i} \omega \boldsymbol{m}_{l}=\gamma \mu_{0}\left(\boldsymbol{M}_{S, l}+\boldsymbol{m}_{l}\right) \times \boldsymbol{H}_{\mathrm{eff}}
$$

with the following form of the local effective field [10-12]:

$$
\boldsymbol{H}_{\mathrm{eff}}=\boldsymbol{H}_{0}+h_{l}+\frac{2}{\mu_{0}}\left(\nabla \cdot \frac{A_{l}}{M_{S, l}^{2}} \nabla\right) m_{l},
$$

where $\boldsymbol{H}_{0}$ is the external field, $\boldsymbol{h}_{l}$ is the dipolar field, $M_{S, l}$ is the saturated magnetization and $A_{l}$ is the local exchange constant. In Eq. (3) we assumed that $M_{0, l} \approx M_{S, l}$. In a one-dimensional discrete approach to the layered composite material, with a carefully defined central layer where the derivation takes place, this field can be expressed as follows:

$$
\begin{aligned}
\boldsymbol{H}_{\mathrm{eff}, l} & =\boldsymbol{H}_{0}+\boldsymbol{h}_{l} \\
& +\frac{2}{\mu_{0} a^{2}}\left[\frac{A_{l+1, l}}{M_{S, l+1}^{2}} \boldsymbol{m}_{l+1}-\left(\frac{A_{l+1, l}}{M_{S, l+1}^{2}}+\frac{A_{l, l-1}}{M_{S, l}^{2}}\right) \boldsymbol{m}_{l}+\frac{A_{l, l-1}}{M_{S, l}^{2}} \boldsymbol{m}_{l-1}\right],
\end{aligned}
$$


where $a$ is the lattice parameter of the composite material, and $A_{l+1, l}$ is the exchange constant linking spins of layers $l$ and $l+1$. To simplify calculations, it is convenient to introduce the concept of local exchange field $A_{l}$. With this local concept, which has been largely used in transfer matrix techniques, the effective field reads

$$
\begin{aligned}
\boldsymbol{H}_{\mathrm{eff}, l} & =\boldsymbol{H}_{0}+\boldsymbol{h}_{l} \\
& +\frac{2}{\mu_{0} a^{2}}\left[\frac{A_{l+1}}{M_{S, l+1}^{2}} \boldsymbol{m}_{l+1}-\left(\frac{A_{l+1}}{M_{S, l+1}^{2}}+\frac{A_{l}}{M_{S, l}^{2}}\right) \boldsymbol{m}_{l}+\frac{A_{l}}{M_{S, l}^{2}} \boldsymbol{m}_{l-1}\right] .
\end{aligned}
$$

Then, it is useful to introduce the effective local parameter

$$
J_{l}=\frac{2 A_{l}}{\mu_{0} a^{2} M_{S, l}^{2}}
$$

to obtain a more compact expression of the effective field

$$
\boldsymbol{H}_{\mathrm{eff}, \mathrm{l}}=\boldsymbol{H}_{0}+h_{l}+\left[J_{l+1} \boldsymbol{m}_{l+1}-\left(J_{l+1}+J_{l}\right) \boldsymbol{m}_{l}+J_{l} \boldsymbol{m}_{l-1}\right] .
$$

According to this expression, the linearized equation of motion for magnetization $(3)$ in the case of weak spin excitation reads

$$
\begin{aligned}
& -\mathrm{i} \omega \boldsymbol{m}_{l}=\gamma \mu_{0} \boldsymbol{M}_{S, l} \\
& \quad \times\left[\boldsymbol{h}_{l}+J_{l+1} \boldsymbol{m}_{l+1}-\left(J_{l+1}+J_{l}\right) \boldsymbol{m}_{l}+J_{l} \boldsymbol{m}_{l-1}\right]+\gamma \mu_{0} \boldsymbol{m}_{l} \times \boldsymbol{H}_{0} .
\end{aligned}
$$

Applying the saturating field means that both the static magnetization $\boldsymbol{M}_{S, l}$ and the external field $\boldsymbol{H}_{0}$ are parallel to each other and perpendicular to the plane $P$ of the dynamic magnetization $\boldsymbol{m}_{\boldsymbol{l}}$. Thus, a complex representation of the plane $P$, where all the dynamic magnetization vectors $m_{l}$ lie, enables us to deduce a simple expression of the vector products if we introduce the following definition of complex $m_{l}: m_{l} \equiv m_{l}^{x}+\mathrm{i} m_{l}^{y}$. Then, dividing Eq. (9) by $\gamma \mu_{0} H_{0}$ and using the frequency variable $g$, defined as:

$$
g=\frac{\omega}{\gamma \mu_{0} H_{0}},
$$

and the magnetic stiffness parameter

$$
C_{l}=\frac{M_{S, l}}{H_{0}}
$$

we are led to a simpler equation of motion

$$
C_{l} h_{l}+C_{l} J_{l+1} m_{l+1}+\left[g-C_{l}\left(J_{l+1}+J_{l}\right)-1\right] m_{l}+C_{l} J_{l} m_{l-1}=0 .
$$

The simple resolution of Maxwell's equation

$$
\boldsymbol{\nabla} \cdot\left(h_{l}+m_{l}\right) \approx h_{l+1}-h_{l}+m_{l+1}-m_{l}=0
$$

leads us to write quite classically $h_{l}=-m_{l}$ which reduces the long ranged dipolar effect to a short ranged one. Breaking the symmetry, (12) and (13) can be read together in a regressive way, in order to define a transfer matrix: 


$$
m_{l-1}=\frac{m_{l}}{J_{l}}+\frac{1+C_{l}\left(J_{l}+J_{l+1}\right)-g}{C_{l} J_{l}} m_{l}-\frac{J_{l+1}}{J_{l}} m_{l+1}
$$

and

$$
\left(\begin{array}{c}
m_{l-1} \\
m_{l}
\end{array}\right)=\left(\begin{array}{cc}
\frac{1+C_{l}\left(1+J_{l}+J_{l+1}\right)-g}{C_{l} J_{l}} & -\frac{J_{l+1}}{J_{l}} \\
1 & 0
\end{array}\right)\left(\begin{array}{c}
m_{l} \\
m_{l+1}
\end{array}\right) .
$$

When dealing with a composite sample made of two materials $\mathrm{A}$ and $\mathrm{B}$, with different exchange constant and magnetic stiffness values, four transfer matrices must be defined: $\widehat{t}_{\mathrm{A}}, \widehat{t}_{\mathrm{B}}, \widehat{t}_{\mathrm{AB}}$, and $\widehat{t}_{\mathrm{BA}}$. They are

$$
\begin{array}{ll}
\widehat{t}_{\mathrm{A}}=\left(\begin{array}{cc}
2+\frac{1-g+C_{\mathrm{A}}}{C_{\mathrm{A}} J_{\mathrm{A}}} & -1 \\
1 & 0
\end{array}\right), & \widehat{t}_{\mathrm{B}}=\left(\begin{array}{cc}
2+\frac{1-g+C_{\mathrm{B}}}{C_{\mathrm{B}} J_{\mathrm{B}}} & -1 \\
1 & 0
\end{array}\right), \\
\hat{t}_{\mathrm{AB}}=\left(\begin{array}{cc}
1+\frac{1-g+C_{\mathrm{B}}\left(J_{\mathrm{A}}+1\right)}{C_{\mathrm{B}} J_{\mathrm{B}}} & -\frac{J_{\mathrm{A}}}{J_{\mathrm{B}}} \\
1 & 0
\end{array}\right), & \widehat{t}_{\mathrm{BA}}=\left(\begin{array}{cc}
1+\frac{1-g+C_{\mathrm{A}}\left(J_{\mathrm{B}}+1\right)}{C_{\mathrm{A}} J_{\mathrm{A}}} & -\frac{J_{\mathrm{B}}}{J_{\mathrm{A}}} \\
1 & 0
\end{array}\right) .
\end{array}
$$

Considering the composite material ABABABA, where each block $\mathrm{A}$ is made of $L_{\mathrm{A}}$ layers and each block $\mathrm{B}$ is made of $L_{\mathrm{B}}$ layers (see Fig. 1), the global transfer matrix $\widehat{T}$ is defined as follows:

$$
\widehat{T}=\hat{t}_{\mathrm{A}}^{L_{\mathrm{A}}-1} \cdot \hat{t}_{\mathrm{BA}} \cdot \hat{t}_{\mathrm{B}}^{L_{\mathrm{B}}-1} \cdot \hat{t}_{\mathrm{AB}} \cdot \hat{t}_{\mathrm{A}}^{L_{\mathrm{A}}-1} \cdots \hat{t}_{\mathrm{BA}} \cdot \hat{t}_{\mathrm{B}}^{L_{\mathrm{B}}-1} \cdot \hat{t}_{\mathrm{AB}} \cdot \hat{t}_{\mathrm{A}}^{L_{\mathrm{A}}}
$$

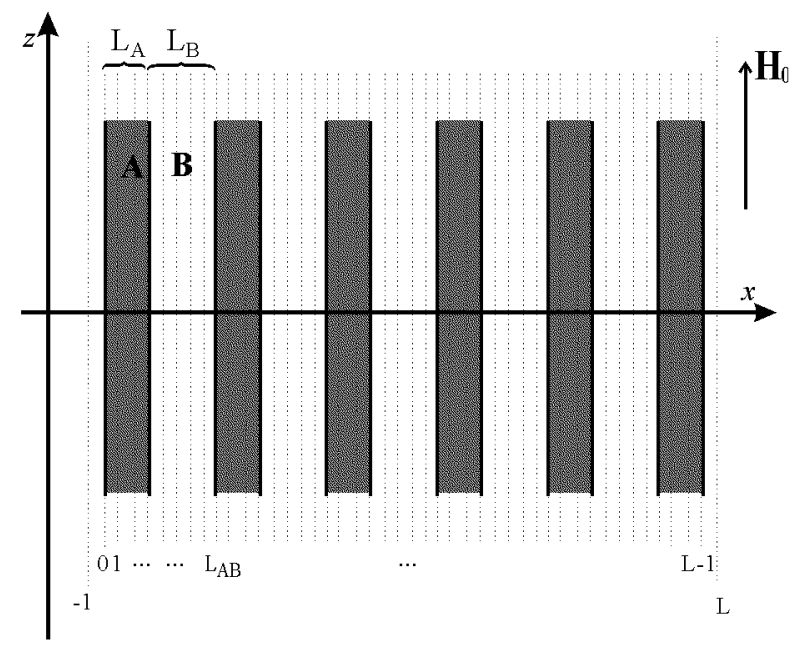

Fig. 1. The model of a multilayer composed of $N_{\mathrm{A}}=6$ sublayers of material $\mathrm{A}$ and $N_{\mathrm{B}}=5$ sublayers of material B. Each sublayer $\mathrm{A}$ or $\mathrm{B}$ contains $L_{\mathrm{A}}$ or $L_{\mathrm{B}}$ planes respectively. $L$ is the total number of planes in the multilayer. 
The boundary conditions are defined by the partial pinning equations involving surface pinning parameters $[20-23] a_{0}$ and $a_{0}^{\prime}$ :

$$
\begin{aligned}
& m_{-1}=a_{0} m_{0}, \\
& m_{L}=a_{0}^{\prime} m_{L-1},
\end{aligned}
$$

where (in the case considered here) the total number $L$ is $4 L_{\mathrm{A}}+3 L_{\mathrm{B}}$. Thus, the characteristic equation reads

$$
\left(1,-a_{0}\right) \widehat{T}\left(\begin{array}{c}
1 \\
a_{0}^{\prime}
\end{array}\right)=0 .
$$

Then, the spin-wave mode profiles can be calculated according to the partial transfer matrix, which is deduced from Eq. (17).

\section{A particular case: transfer matrices without dipolar field}

In spin-wave frequency calculations using the Landau-Lifshitz equations (3), the dipolar field can be neglected for sufficiently high wave vector values $(k>$ $10^{8} \mathrm{~m}^{-1}$ ). Then, the effective field reads

$$
\boldsymbol{H}_{\mathrm{eff}}=\boldsymbol{H}_{0}+\frac{2}{\mu_{0}}\left(\boldsymbol{\nabla} \cdot \frac{A_{l}}{M_{S, l}^{2}} \nabla\right) \boldsymbol{m}_{l},
$$

and the difference form of the Landau-Lifshitz equation is equivalent to Eq. (12):

$$
C_{l} J_{l+1} \boldsymbol{m}_{l+1}+\left[g-C_{l}\left(J_{l+1}+J_{l}\right)-1\right] \boldsymbol{m}_{l}+C_{l} J_{l} \boldsymbol{m}_{l-1}=0
$$

which can be written also in the following matrix form:

$$
\left(\begin{array}{c}
m_{l-1} \\
m_{l}
\end{array}\right)=\left(\begin{array}{cc}
\frac{1+C_{l}\left(J_{l}+J_{l+1}\right)-g}{C_{l} J_{l}} & -\frac{J_{l+1}}{J_{l}} \\
1 & 0
\end{array}\right)\left(\begin{array}{c}
m_{l} \\
m_{l+1}
\end{array}\right) .
$$

For a composite consisting of two different materials, the following four $2 \times 2$ matrices must be defined:

$$
\begin{array}{cc}
\widehat{t}_{\mathrm{A}}=\left(\begin{array}{cc}
2+\frac{1-g}{C_{\mathrm{A}} J_{\mathrm{A}}} & -1 \\
1 & 0
\end{array}\right), & \widehat{t}_{\mathrm{B}}=\left(\begin{array}{cc}
2+\frac{1-g}{C_{\mathrm{B}} J_{\mathrm{B}}} & -1 \\
1 & 0
\end{array}\right), \\
\widehat{t}_{\mathrm{AB}}=\left(\begin{array}{cc}
1+\frac{1-g+C_{\mathrm{B}} J_{\mathrm{A}}}{C_{\mathrm{B}} J_{\mathrm{B}}} & -\frac{J_{\mathrm{A}}}{J_{\mathrm{B}}} \\
1 & 0
\end{array}\right), & \widehat{t}_{\mathrm{BA}}=\left(\begin{array}{cc}
1+\frac{1-g+C_{\mathrm{A}} J_{\mathrm{B}}}{C_{\mathrm{A}} J_{\mathrm{A}}} & -\frac{J_{\mathrm{B}}}{J_{\mathrm{A}}} \\
1 & 0
\end{array}\right) .
\end{array}
$$

For a composite structure: ABABABA, with boundary conditions (18), (19), the characteristic equation reads (as in the preceding section)

$$
\left(1,-a_{0}\right) \widehat{T}\left(\begin{array}{c}
1 \\
a_{0}^{\prime}
\end{array}\right)=0
$$

where $\hat{T}$ is still defined by (17), but the factor matrices are now specified by (24). Below, the results of calculations based on Eqs. (20) and (25) are compared, in order to estimate the effect of dipolar fields on the spin-wave spectrum. 


\section{Effect of dipolar interactions on the spin-wave spectrum}

A multilayer investigated here is represented as a one-dimensional lattice composed of $L$ planes (see Fig. 1). As we will see later on, the accuracy of our numerical results obtained from the characteristic Eqs. (20) and (25) will essentially depend on the number $L$ assumed; the criterion for satisfactory convergence we use in our computations will be discussed in detail in Sec. 7.

First, we examine the effect of long-ranged dipolar interactions on the spin-wave modes for a magnetic multilayer. However, in our study, the effect of dipolar interactions on spin waves is not related to the existence of surface magnetostatic excitations [24-31]. We simply compare the magnon spectra calculated according to (20), where both dipolar and exchange interactions are considered, with those obtained from (25), where we take into account exchange interactions only; we pay the special attention to the shape of spin-wave mode profiles. This comparison will determine conditions for which the dipolar field can be neglected to simplify further calculations.

We find that in the considered multilayer, with the "lattice" constant $\left(L_{\mathrm{A}}+L_{\mathrm{B}}\right) a=100 \AA$, dipolar interactions modify the exchange spin-wave energy spectrum causing only minor shifts of spin-wave branches towards higher frequency values (see Figs. 2a and b). For a strong magnetization contrast (Fig. 2a), this shift depends on the filling fraction defined as the thickness ratio of the layer $A$ to the bilayer AB (regarded as the unit cell)

$$
f=\frac{L_{\mathrm{A}}}{L_{\mathrm{A}}+L_{\mathrm{B}}} .
$$

For a composite with component materials differing in their exchange constants only (Fig. 2b), the shift is insensitive to the filling fraction.

We also notice an effect of dipolar field on the lowest magnon mode profiles. Figure 3 shows profiles of the first three exchange-dipolar modes (thick line) and the corresponding pure exchange modes (thin line), for a system composed of six Fe layers and five yttrium-iron garnet (YIG) layers. Since it is seen that the effect of dipolar field becomes negligible for higher frequencies (higher modes), we will confine our interest to the lowest mode. As shown in Fig. 3, in the sublayer having larger spontaneous magnetization value $\left(M_{S_{\mathrm{Fe}}}=1.752 \times 10^{6} \mathrm{~A} \mathrm{~m}^{-1}\right)$, the dipolar field deforms the previously linear profile towards a standing wave with an incomplete sinusoidal arch (with partially pinned spins on interfaces). The interface pinning seems to be due to the relatively high magnetization value of iron with respect to that of YIG $\left(M_{S_{\mathrm{Fe}}} / M_{S_{\mathrm{YIG}}} \approx 9.0\right)$. Furthermore, we notice that these deformations are more significant for the internal iron layers than for the external ones. In our opinion, this is due to the larger distance of the former from the multilayer surface, where the spins are assumed to be completely pinned. Figure 4 shows the lowest mode profiles for three (Fe/YIG) multilayer composites differing in number of unit cells (Fig. 4a), as well as for the composite with inverse sublayer 

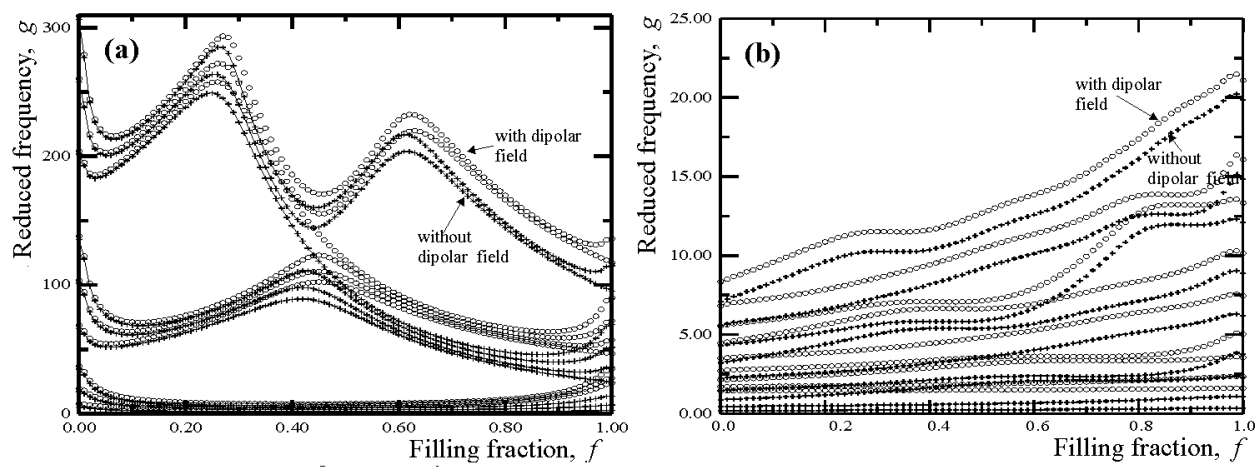

Fig. 2. Spin-wave mode energy spectrum vs. filling fraction $f$, computed for two cases: with dipolar field included (empty circles) or neglected (crosses): (a) multilayer $\mathrm{Fe} / \mathrm{YIG} / \mathrm{Fe} / \mathrm{YIG} / \mathrm{Fe} / \mathrm{YIG} / \mathrm{Fe}$ with $M_{S_{\mathrm{Fe}}}=1.752 \times 10^{6} \mathrm{~A} \mathrm{~m}^{-1}, A_{\mathrm{Fe}}=$ $2.1 \times 10^{-11} \mathrm{~J} \mathrm{~m}^{-1}$ and $M_{S_{\mathrm{YIG}}}=0.194 \times 10^{6} \mathrm{~A} \mathrm{~m}^{-1}, A_{\mathrm{YIG}}=0.4 \times 10^{-11} \mathrm{~J} \mathrm{~m}^{-1}$ - the case of a multilayer with strong magnetization contrast; (b) multilayer A/B/A/B/A/B/A with $M_{S_{\mathrm{A}}}=M_{S_{\mathrm{B}}}=1.0 \times 10^{6} \mathrm{~A} \mathrm{~m}^{-1}$ and $A_{\mathrm{B}}=0.4 \times 10^{-11} \mathrm{~J} \mathrm{~m}^{-1}, A_{\mathrm{A}}=$ $2.1 \times 10^{-11} \mathrm{~J} \mathrm{~m}^{-1}-$ a multilayer without magnetization contrast.

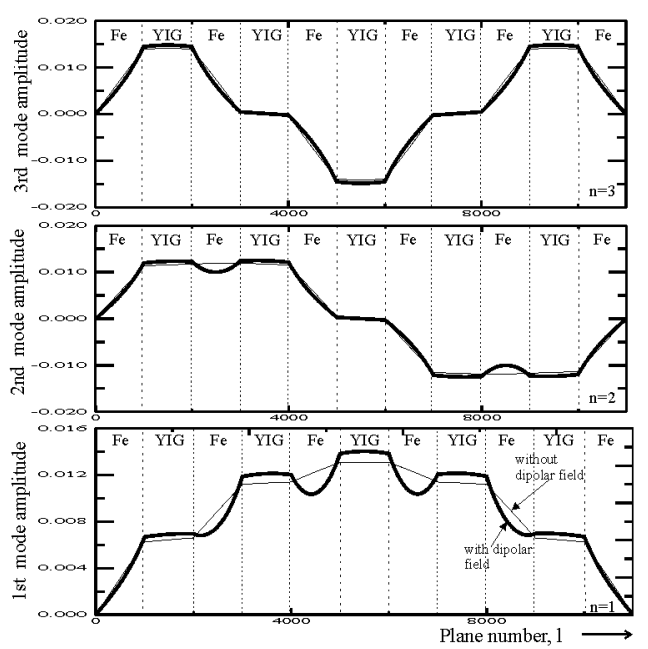

Fig. 3. The 1st, 2nd, and 3rd normalized mode amplitude profiles for exchange-dipolar spin-wave modes in a Fe/YIG multilayer (thick lines); for comparison, profiles of pure exchange modes are also shown (thin lines).

arrangement (YIG/Fe) (Fig. 4b). The shapes of these profiles clearly indicate that the effect of surface conditions on excitations within the multilayer is the strongest for the external sublayers and weakens for the internal ones.

Since further on we will focus on high energy states, for which the effect of dipolar field is minor, in the following sections we take into account exchange field 

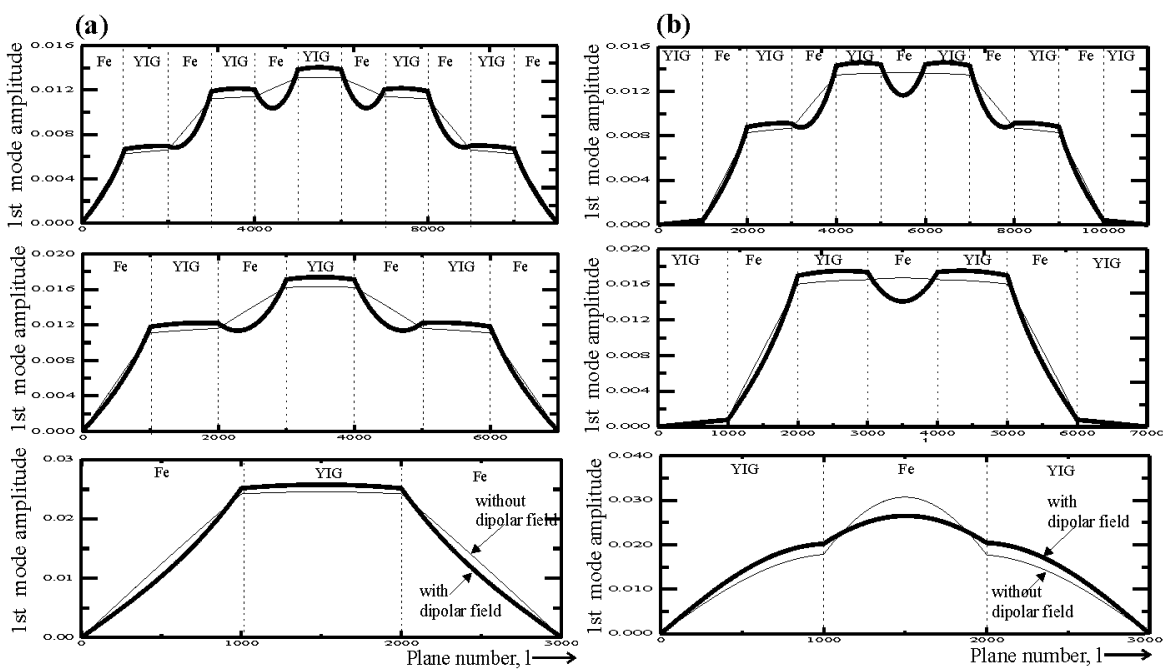

Fig. 4. The 1st mode normalized amplitude profile for (a) Fe/YIG and (b) YIG/Fe multilayers, calculated with dipolar field included (thick lines) or neglected (thin lines), for different numbers of unit cells assumed in the multilayer.

only. The excitations studied below propagate perpendicularly to the interfaces and to the external static magnetic field (in this study we assume $\mu_{0} H_{0}=0.1 \mathrm{~T}$ ).

\section{Spin-wave mode profiles in a multilayer}

Excitations in a multilayer sample generally behave like those in a homogeneous film. An example is shown in Fig. 3: for a symmetrical multilayer with surface spins pinned, the modes form alternately symmetrical and antisymmetrical standing waves with nodes on the surfaces, exactly as for a homogeneous film. The difference is that the multilayer profiles do not vary homogeneously inside the sample, due to perturbations caused by interfaces. Now we are going to study the effect of interfaces (as well as that of surface conditions) on the shape of mode profiles in a multilayer.

Figure 5 shows variations of the first mode profile for different filling fraction values. For all the three filling fraction values $(f=0.1, f=0.5$, and $f=0.9)$, the profiles preserve the general form of the first harmonic. It should be noticed that all the sublayers show asymmetry in their partial profiles, with their amplitudes being the lowest on the side closer to the surface. This effect is stronger for iron layers, due to their high magnetization value, but it also occurs (although is much weaker) for YIG sublayers (cf. cases $f=0.1$ and $f=0.9$ in Fig. 5); the asymmetry disappears in the central zone. This type of behavior is also observed for all the higher modes, though the effect decreases as frequency increases.

Figure 6 shows profiles of the 1st, 4th, and 7th modes belonging, respectively, to the lowest branches of the first three bands of the investigated composite, for 


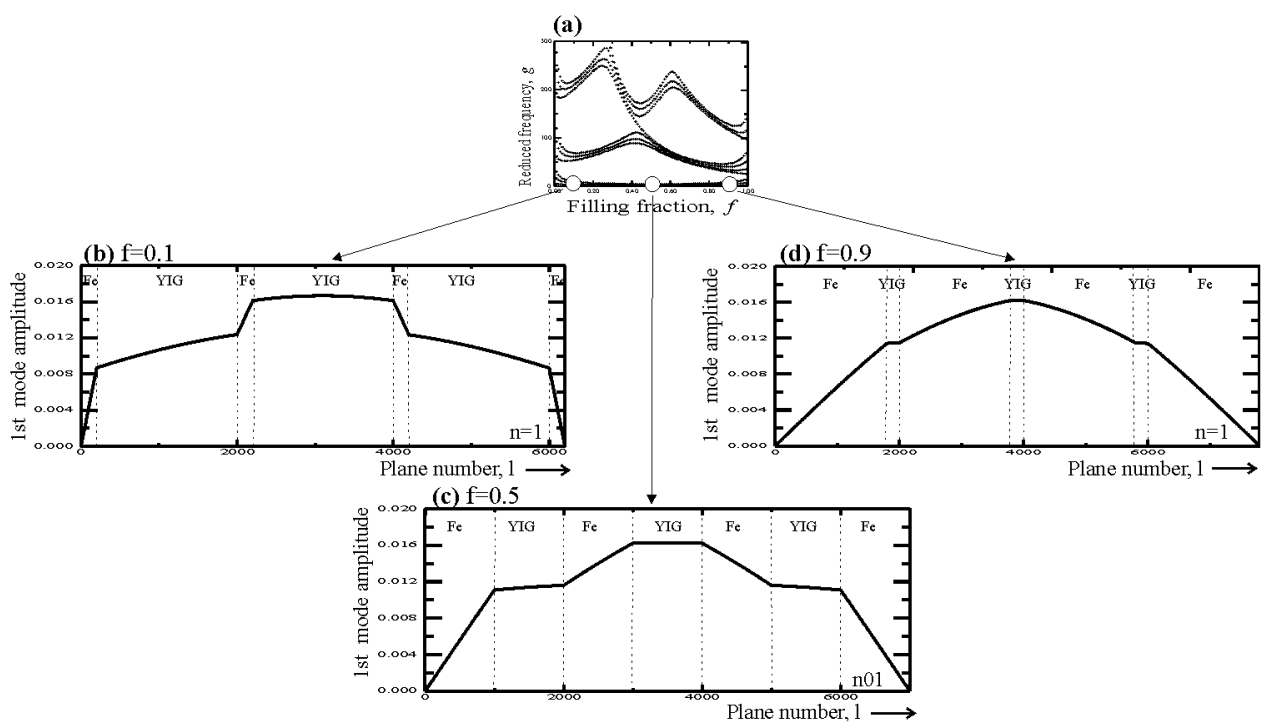

Fig. 5. The 1st mode normalized amplitude profile across the multilayer YIG/Fe for different values of the filling fraction: (b) $f=0.1$, (c) $f=0.5$, (d) $f=0.9$; (a) the respective spin-wave mode spectrum, with open circles indicating positions of the depicted lowest energy branch modes.

two filling fraction values: (a) $f=0.1$, in which case iron is the predominating material, and (b) $f=0.9$, with garnet (YIG) predominating. The lowest $(n=1)$ mode profile (the same as in Fig. 5) is composed of more or less linear segments corresponding to particular sublayers. For $n=4$ (the first branch of the second band), the mode profile comprises sinusoidal arches in the predominant material, the interface amplitude being maximum for YIG (if $f=0.1$ ) and minimum (close to zero) for Fe sublayers (if $f=0.9$ ). The lowest mode of the third band, $n=7$, reveals similar features. This may mean that the interface spins in Fe sublayers (i.e. those in contact with garnet) are strongly pinned, while the YIG interface spins (being in contact with iron) behave like unpinned ones.

The results presented below and obtained in the so-called independent layers approximation give a deeper insight into the profile variation rules of the multilayer.

\section{Approximate treatments}

Numerical calculations of the frequency spectrum as a function of the relative concentration $g\left(L_{\mathrm{A}} /\left(L_{\mathrm{A}}+L_{\mathrm{B}}\right)\right)$ indicate occurrence of forbidden gaps. Thus, the band limits can be found from approximate treatment. Two simplified models can be applied, according to the case: when the contrast is strong, the component materials can be regarded as independent, and when the contrast is weak, an effective medium can be considered in place of the non-homogeneous material. 
(a) $\mathrm{f}=0.1$
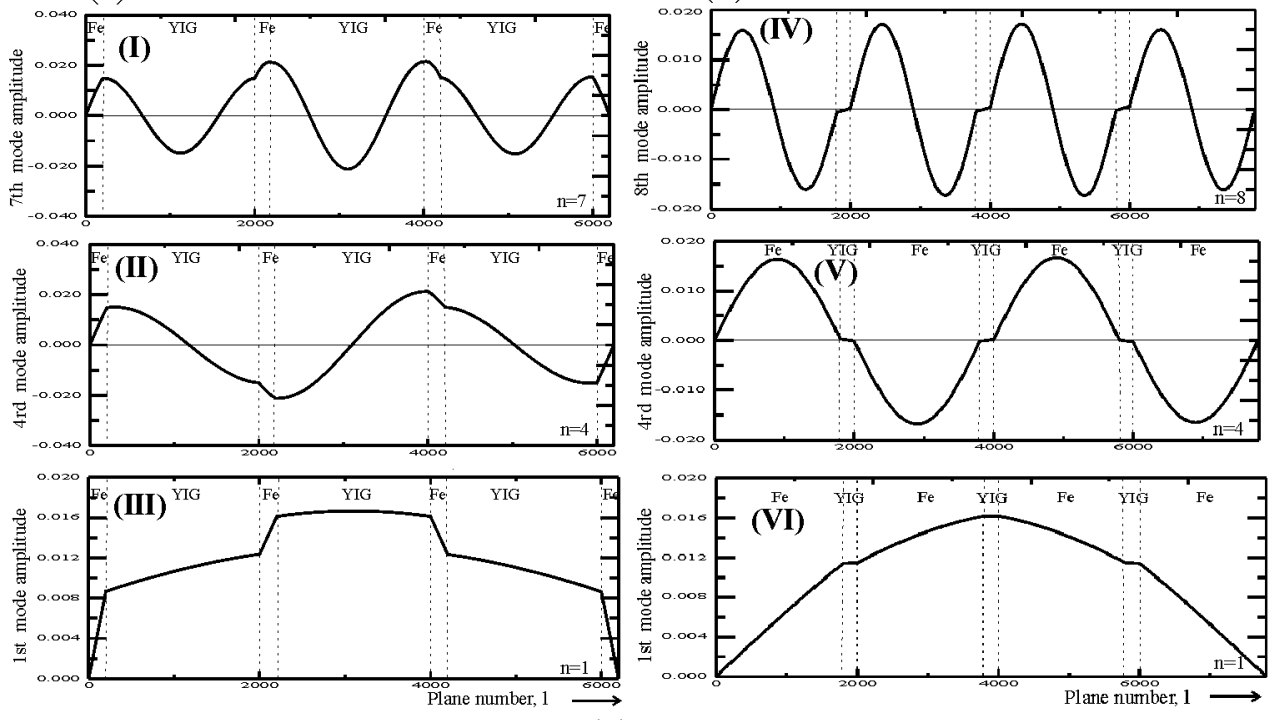

(c)
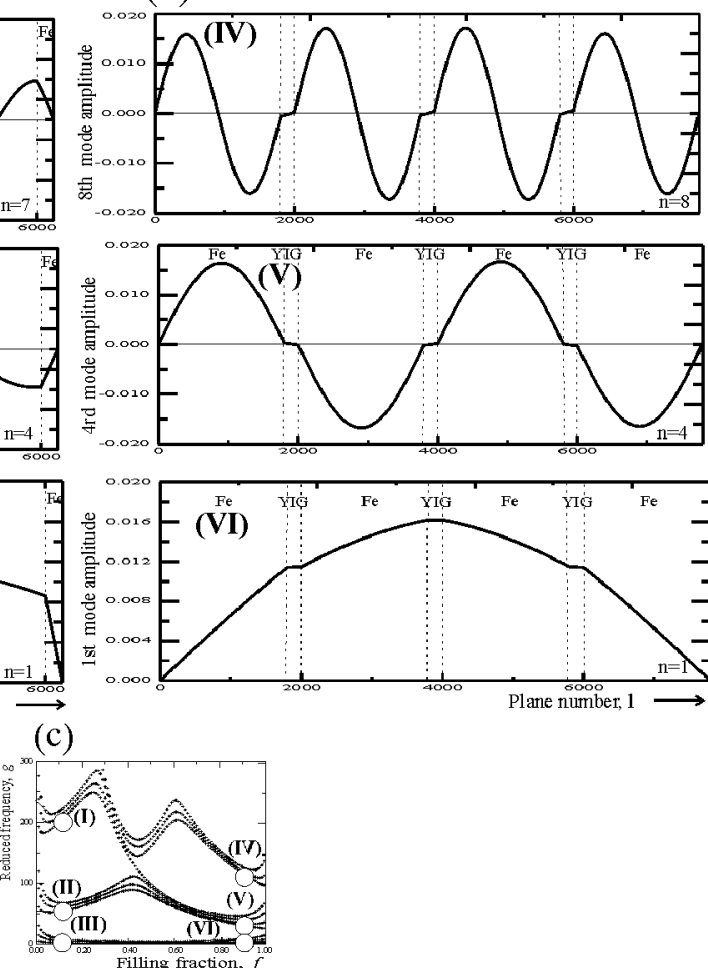

Fig. 6. (a) Normalized profiles of the 1st, 4th, and 7th spin-wave modes of the Fe/YIG multilayer obtained for the filling fraction value $f=0.1$ [branches of the respective spin-wave mode spectrum, I-III, are given in (c)]. (b) Normalized profiles of the 1st, $4 \mathrm{th}$, and 8th spin-wave modes of the Fe/YIG multilayer obtained for the filling fraction value $f=0.9$ [branches of the respective spin-wave mode spectrum, IV-VI, are given in $(c)]$.

\subsection{Strong contrast}

At least in the case of a strong contrast, it is tempting to relate these bands to the spin-wave propagation within a single layer with effective boundary conditions. This is the independent layer model. For this model, the eigenvalue $s$ of the propagation through one layer of material A reads

$$
\operatorname{det}\left(\widehat{t}_{\mathrm{A}}-s \widehat{I}\right)=0
$$

i.e.

$$
s^{2}-\left(2+\frac{1-g}{C_{\mathrm{A}} J_{\mathrm{A}}}\right) s+1=0 .
$$

Thus, if $g>1$, which defines a limiting value of the frequency spectrum, the eigenmode is oscillating with $s=\mathrm{e}^{ \pm \mathrm{i} \theta}$, where $\cos (\theta)=1-\frac{g-1}{2 C_{\mathrm{A}} J_{\mathrm{A}}}$ and $\theta$ is the phase shift between successive layers. The basic assumption for propagation within one 
film A of thickness $a L_{\mathrm{A}}$ leads to an integer number $k$ of sinusoidal arches within this film: $\theta=k \pi / L_{\mathrm{A}}$. More accurately, partial pinning at the boundary will lead to real numbers $k$, which can be classified by the previous relation since successive modes differ by about one arch. With this simple model, the frequency spectrum for A follows:

$$
g_{\mathrm{A}}=1+4 C_{\mathrm{A}} J_{\mathrm{A}} \sin ^{2}\left(\frac{k \pi}{2 L_{\mathrm{A}}}\right) \approx 1+C_{\mathrm{A}} J_{\mathrm{A}}\left(\frac{k \pi}{L_{\mathrm{A}}}\right)^{2},
$$

where the approximation assumes that the film A is thick enough. Quite similarly, the frequency spectrum for a film $B$ reads

$$
g_{\mathrm{B}}=1+4 C_{\mathrm{B}} J_{\mathrm{B}} \sin ^{2}\left(\frac{k \pi}{2 L_{\mathrm{B}}}\right) \approx 1+C_{\mathrm{B}} J_{\mathrm{B}}\left(\frac{k \pi}{L_{\mathrm{B}}}\right)^{2} .
$$

The frequency spectrum of the composite material results from the superposition of two hyperbolic-like functions observed in numerical spectra (see Fig. 7a).

Figure $7 \mathrm{~b}$ shows the reduced spin-wave mode frequencies (crosses) as a function of the filling fraction $f$. The calculations were made for a symmetrical mul-
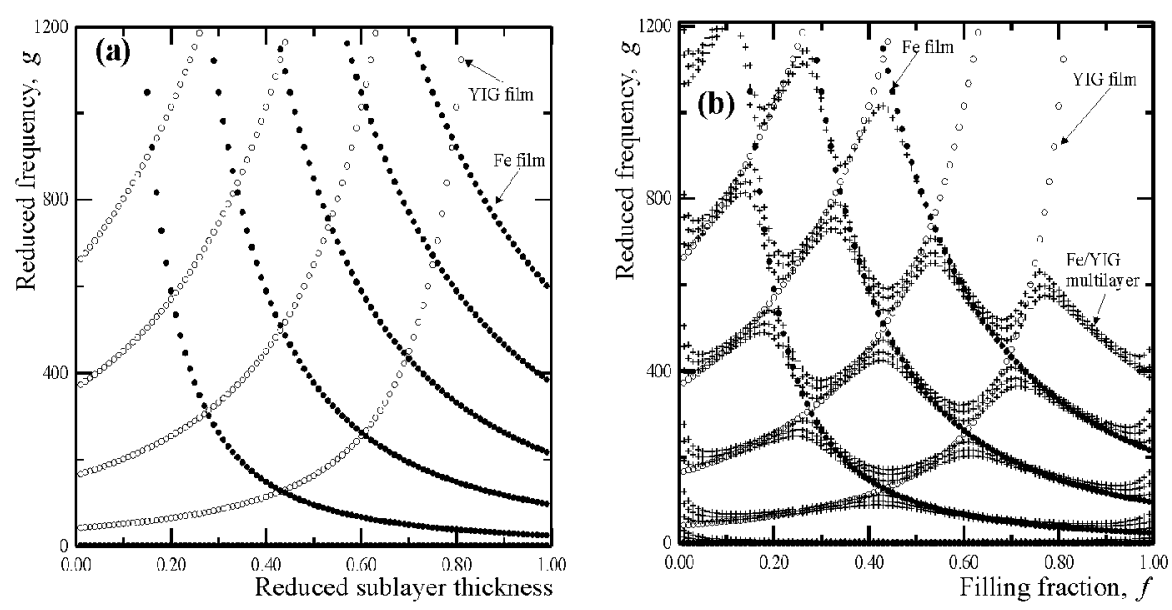

Fig. 7. (a) Frequency branches of spin-wave modes in independent sublayers Fe (full circles) and YIG (empty circles); the branches are depicted as functions of the respective reduced sublayer thickness (filling fraction, $f$ ). (b) Spin-wave mode branches as a function of the filling fraction for the multilayer $\mathrm{Fe} / \mathrm{YIG} / \mathrm{Fe} / \mathrm{YIG} / \mathrm{Fe} / \mathrm{YIG} / \mathrm{Fe}$ (calculated points are marked with crosses). As a guide for eyes, branches of independent entities are also shown: full circles correspond to an Fe sublayer, and empty ones to a YIG sublayer. 
tilayer composed of four layers of material A $(\mathrm{Fe})$ and three layers of material B (YIG) (structure ABABABA). The contrasts of spontaneous magnetization and exchange constant between iron and garnet were 9.03 and 5.25 respectively, so the studied case is that of a strong magnetization contrast. Energy gaps occur between bands, but only the first gap (between the first and the second band) exists in the whole range of the filling fraction. The others are oscillating, with "repulsion" taking place in some points: between the second and the third band, the repulsion occurs for one filling fraction value only $(f \approx 0.43)$, while the third and the fourth bands are repulsed in two points $(f \approx 0.28$ and $f \approx 0.60)$, the following two bands - in three points, and generally, the number of repulsion points increases by one as the band number increases. In order to explain the reasons of this repulsion effect, relations (29) (filled circles) and (30) (empty circles) are plotted in Fig. 7b, showing spin-wave mode branches in independent layers of iron and garnet as a function of reduced thickness (these branches have been shown separately in Fig. 7a); four hyperbolic-like curves were plotted, corresponding to the lowest values of $k=0,1,2$ and 3 . These curves indicate the general distribution of the multilayer bands and allow to find all the energy gaps with quite good accuracy. Intersection points of two curves belonging to different families are degeneration points of the repulsed bands; hence, the energy gap disappearing condition is $g_{\mathrm{A}}=g_{\mathrm{B}}$.

We will now go back to the analysis of the profile shapes which we started in Sec. 5. The sinusoidal arches and their multiples in the profiles shown in Fig. 6a

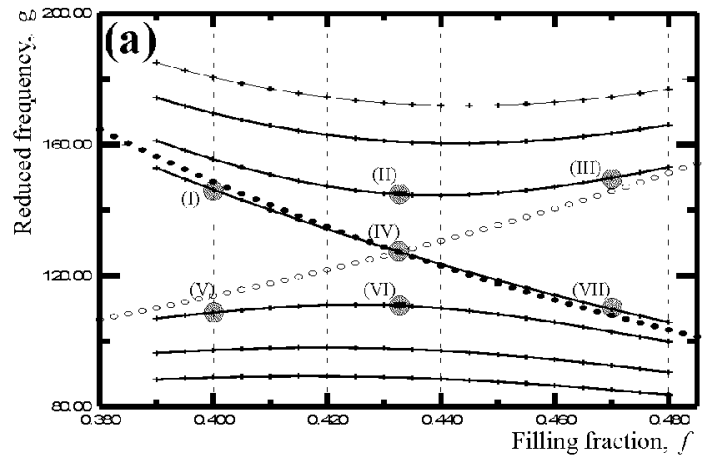

Fig. 8. Normalized profiles of spin-wave modes across the Fe/YIG multilayer in the frequency region where the 2nd bands of independent YIG and Fe layers cross (see Fig. 7); note that these bands (related to independent sublayers!) are indicated here (as in Fig. 7) with full circles for Fe and empty ones for YIG. (a) The distribution of multilayer frequency branches (solid lines) in the region of a repulsion, $f \in(0.38,0.48)$; (b) normalized profiles corresponding to seven points indicated with large circles on the multilayer branches in graph (a) (see next page). 

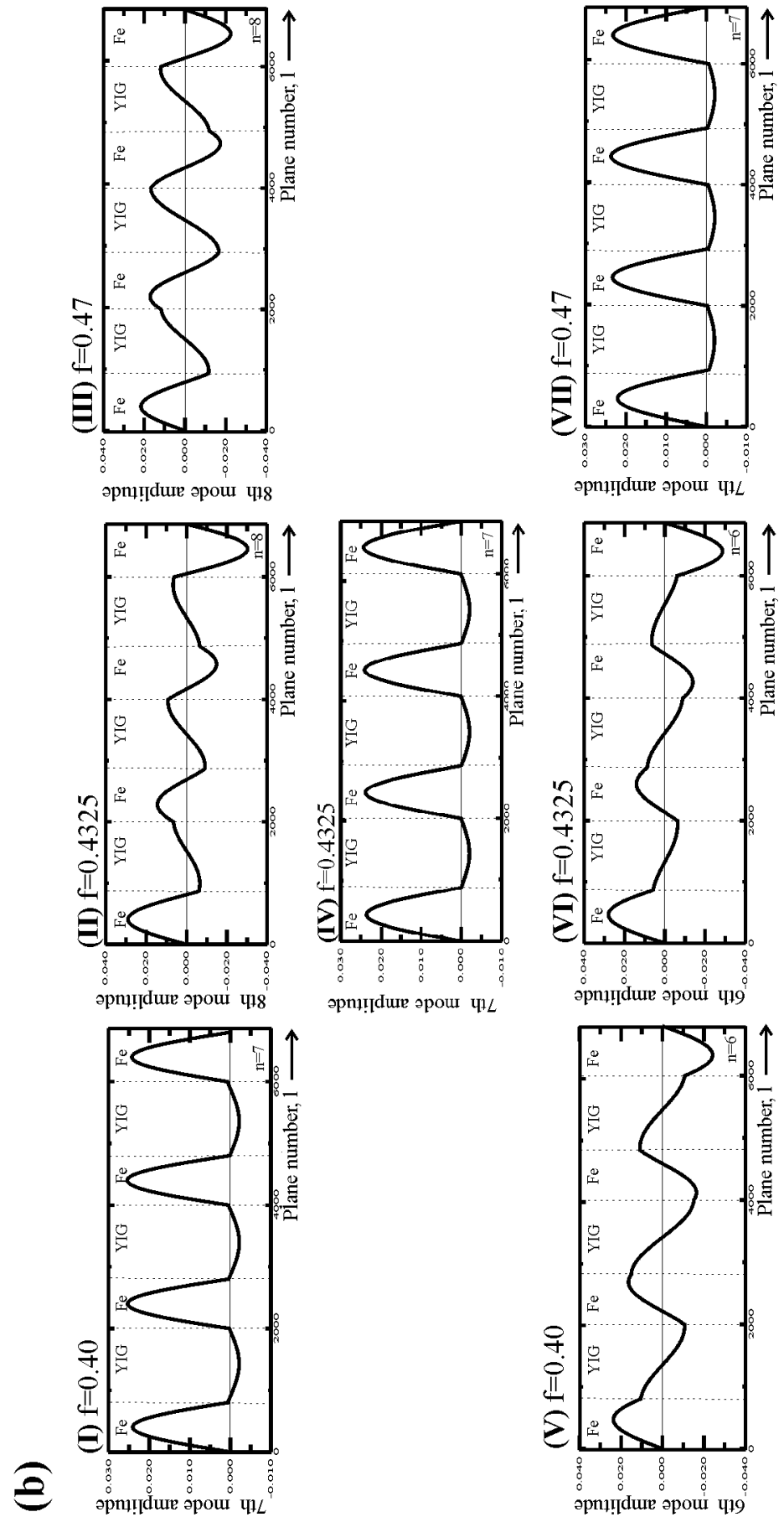
and $\mathrm{b}$ can now be easily interpreted. In Fig. $7 \mathrm{~b}$ the filled-circle branches correspond to excitation frequencies in an independent Fe layer, so the profiles obtained for $f=0.9$ and shown in Fig. $6 \mathrm{~b}$ are harmonics in the Fe sublayers. Similarly, the modes obtained for $f=0.1$ and shown in Fig. 6a correspond to frequencies in a YIG layer (empty circles in Fig. 7a), so they have a form of sinusoidal arches with antinodes on YIG layer interfaces. We will now examine how the mode profiles behave in the vicinity of a repulsion point, e.g. near the intersection of the second and the third band (i.e. for the filling fraction close to $f=0.4325$, corresponding to the intersection point for modes of independent Fe and YIG layers for $k=1$, see Fig. 7a). Figure $8 \mathrm{a}$ shows a fragment of the spectrum depicted in Fig. $7 \mathrm{~b}$, for the filling fraction ranging from 0.38 to 0.48 , with branches from the second and the third bands only. For points marked with shaded circles, profiles were calculated and shown in Fig. 8b. Points I, IV, and VII belong to the energy branch passing from the third band to the second one. This branch coincides with the frequency curve for an independent Fe layer, plotted according to (29) for $k=1$ (filled circles), so (see Fig. 8b) these profiles comprise sinusoidal arches in Fe sublayers, showing no significant variation with $f$. On the contrary, in points V, IV, and III, being close to the frequency curve for an independent YIG layer (empty circles) and belonging to modes $n=6,7$, and 8 respectively, the sinusoidal arches show qualitative differences for different branches in all the garnet sublayers. In the iron sublayer profiles, sinusoidal arches incomplete in point $\mathrm{V}$ become exact sinusoidal arches in point IV, to surpass a single arch in point III.

We now proceed to study the multilayer energy band structure as a function of the exchange and magnetization contrasts between the component materials. For example, let us look for the relation between the spin-wave spectrum and the exchange constant of only one component material, e.g. sublayer A (the other parameters being fixed); two families of straight lines are obtained from (29) and (30). One family comprises lines independent of $A_{\mathrm{A}}$ (see (30)), with increasing interval between the successive lines, given by

$$
J_{\mathrm{B}} \pi^{2} / L_{\mathrm{B}}^{2}\left(k_{i+1}^{2}-k_{i}^{2}\right)
$$

where $k_{i}$ and $k_{i+1}$ are numbers of neighboring lines. The other family is obtained from (29) and comprises straight lines passing through point $C_{\mathrm{A}}$ for $A_{\mathrm{A}}=0$, with increasing slope (Fig. 9). Intersection points for these two families of straight lines determine the multilayer energy band converging points. Similarly, we can analyze the spin-wave spectrum as a function of spontaneous magnetization contrast (Fig. 10): approximate formulae give a family of horizontal straight lines and a family of hyperbolic curves proportional to $M_{S}^{-1}$.

The foregoing reasoning applies to multilayers with a strong contrast between the component materials. In this case, as we have seen, many dispersion features of the system can be deduced from the spectrum of independent layers of materials A (29) and B (30). Now we will proceed to study multilayers composed of materials which differ only slightly in their magnetization and exchange constant values. 


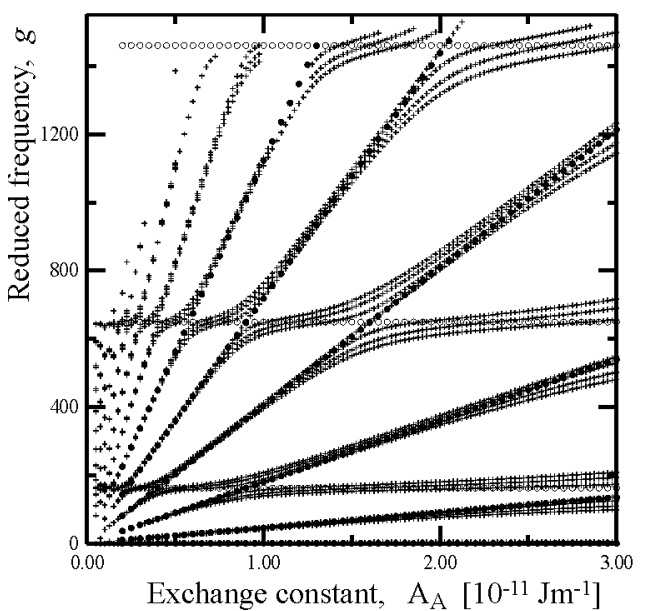

Fig. 9. Frequencies of spin wave modes vs. exchange constant $A_{\mathrm{A}}$ for the multilayer A/YIG/A/YIG/A/YIG/A with magnetization $M_{S_{\mathrm{A}}}=1.752 \times 10^{6} \mathrm{~A} \mathrm{~m}^{-1}$; calculated points are marked with crosses. For comparison, frequencies of the independent $A$ or YIG sublayers are also shown (full circles or empty ones, respectively).

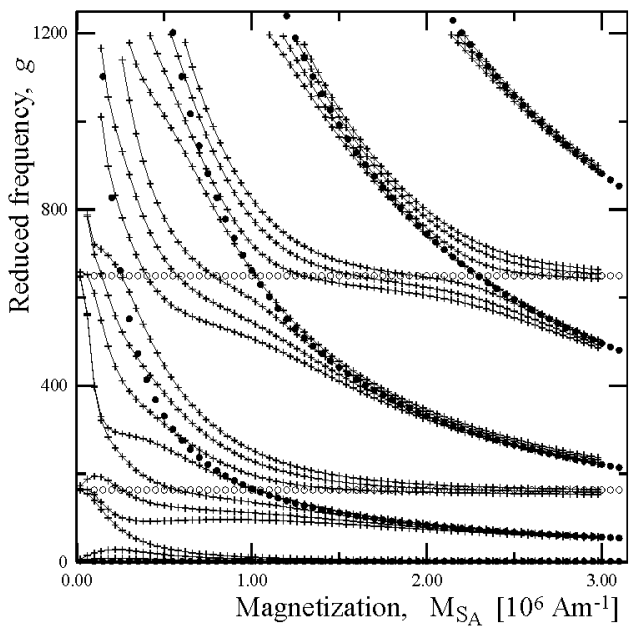

Fig. 10. Frequencies of spin-wave modes vs. magnetization $M_{S_{\mathrm{A}}}$ for the multilayer A/YIG/A/YIG/A/YIG/A with exchange constant $A_{\mathrm{A}}=2.1 \times 10^{-11} \mathrm{~J} \mathrm{~m}^{-1}$; calculated points are marked with crosses. For comparison, frequencies of the independent $A$ or YIG sublayers are also shown (full circles or empty ones, respectively).

\subsection{Weak contrast}

In the case of a weak contrast, a classical approximation applied to such non-homogeneous materials consists in defining a homogeneous average effective medium with effective parameters: 


$$
\begin{aligned}
& C_{\mathrm{E}}=\frac{L_{\mathrm{A}} C_{\mathrm{A}}+L_{\mathrm{B}} C_{\mathrm{B}}}{L_{\mathrm{A}}+L_{\mathrm{B}}}, \\
& J_{\mathrm{E}}=\frac{L_{\mathrm{A}} J_{\mathrm{A}}+L_{\mathrm{B}} J_{\mathrm{B}}}{L_{\mathrm{A}}+L_{\mathrm{B}}} .
\end{aligned}
$$

Then, the spin wave extends over the whole sample and the standard selection of modes gives

$$
\Theta=\frac{k \pi}{L_{\mathrm{A}} N_{\mathrm{A}}+L_{\mathrm{B}} N_{\mathrm{B}}},
$$

where $N_{\mathrm{A}}$ and $N_{\mathrm{B}}$ are the numbers of blocks $\mathrm{A}$ and $\mathrm{B}$, respectively. In the case of a symmetric arrangement of blocks, $N_{\mathrm{A}}=N_{\mathrm{B}} \pm 1$. The foregoing averaging in (32) assumes a very large number of blocks and neglects the difference between $N_{\mathrm{A}}$ and $N_{\mathrm{B}}$. For this effective medium, the previously derived formulae for eigenfrequencies read

$g_{\mathrm{E}}=1+4 C_{\mathrm{E}} J_{\mathrm{E}} \sin ^{2} \frac{k \pi}{2\left(L_{\mathrm{A}} N_{\mathrm{A}}+L_{\mathrm{B}} N_{\mathrm{B}}\right)} \approx 1+C_{\mathrm{E}} J_{\mathrm{E}}\left(\frac{k \pi}{L_{\mathrm{A}} N_{\mathrm{A}}+L_{\mathrm{B}} N_{\mathrm{B}}}\right)^{2}$.

Curves plotted according to this equation are shown in Fig. 11. For comparison, the frequency as a function of the filling fraction is shown there too, for a multilayer composed of four layers of material A and three layers of material B

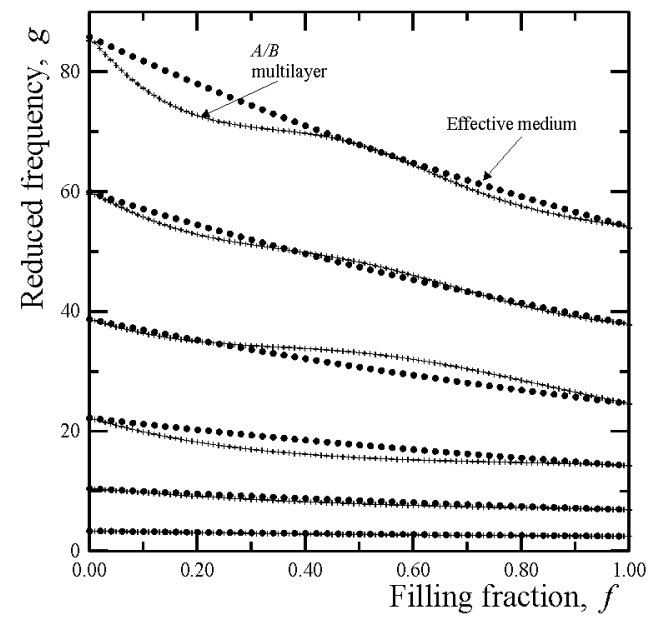

Fig. 11. Frequency branches of spin-wave modes obtained in the effective medium approximation (full circles) compared with those calculated for a multilayer $\mathrm{A} / \mathrm{B} / \mathrm{A} / \mathrm{B} / \mathrm{A} / \mathrm{B} / \mathrm{A}$ (crosses); values assumed: $A_{\mathrm{A}}=2.1 \times 10^{-11} \mathrm{~J} \mathrm{~m}^{-1}, M_{S_{\mathrm{A}}}=$ $1.752 \times 10^{6} \mathrm{~A} \mathrm{~m}^{-1}$, and $A_{\mathrm{B}}=1.4 \times 10^{-11} \mathrm{~J} \mathrm{~m}^{-1}, M_{S_{\mathrm{B}}}=1.5 \times 10^{6} \mathrm{~A} \mathrm{~m}^{-1}$ (weak contrast case). 
(ABABABA). The magnetization and exchange contrasts between the component materials are small, amounting to 1.34 and 1.50 respectively. As expected, $g_{\mathrm{E}}(f)$ is an almost linear function (like for a homogeneous material), decreasing monotonically as the filling fraction increases, and the multilayer spectrum branches oscillate around the effective mean values. Clearly, the fitting is very good and improves as the contrast decreases. Therefore, the effective model is useful in providing rough information on the distribution of multilayer energy branches, and can be applied in spin-wave spectrum analysis for composites with small magnetization and exchange contrasts.

\section{Remarks on the computation convergence by finite difference method}

Figure 12 shows the first and the third mode frequencies, calculated according to (25); a sublayer is divided into planes, the number of which is a variable quantity; the numbers of planes in materials $\mathrm{A}$ and $\mathrm{B}$ are assumed to be equal, i.e. $L_{\mathrm{A}}=L_{\mathrm{B}}$. The multilayer structure of the considered system is $\mathrm{A} / \mathrm{B} / \mathrm{A}$, and the constituent materials have the following parameters: $M_{S_{\mathrm{A}}}=M_{S_{\mathrm{B}}}=$ $1.752 \times 10^{6} \mathrm{~A} \mathrm{~m}^{-1}, A_{\mathrm{A}}=2.1 \times 10^{-11} \mathrm{~J} \mathrm{~m}^{-1}$ and $A_{\mathrm{B}}=1.0 \times 10^{-11} \mathrm{~J} \mathrm{~m}^{-1}$. Figure 12 clearly indicates that as the discrete "lattice" becomes denser, the calculated frequency values become more accurate, asymptotically approaching the eigenfrequency corresponding to $L_{\mathrm{A}} \rightarrow \infty$. This result becomes understandable once we realize that the idea of the finite difference method is based on replacing of the derivatives (in the equation of motion (3)) with difference terms; therefore,
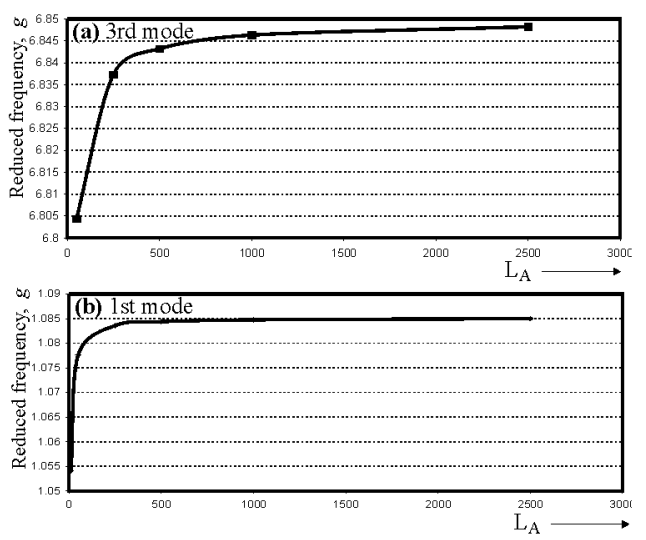

Fig. 12. The frequency convergence test for the 1st mode (b) and the 3rd mode (a); the computed frequency increases with the number of planes $L_{\mathrm{A}}$ assumed in a sublayer A for a trilayer A/B/A with $A_{\mathrm{A}}=2.1 \times 10^{-11} \mathrm{~J} \mathrm{~m}^{-1}, M_{S_{\mathrm{A}}}=M_{S_{\mathrm{B}}}=1.752 \times 10^{6} \mathrm{~A} \mathrm{~m}^{-1}$ and $A_{\mathrm{B}}=1.0 \times 10^{-11} \mathrm{~J} \mathrm{~m}^{-1}$. A and $\mathrm{B}$ sublayers are assumed to have equal thickness $\left(L_{\mathrm{A}}=L_{\mathrm{B}}\right)$. 
the smaller the assumed distance $a$ between neighboring planes, the more the difference term $\frac{m_{l+1}-m_{l}}{a}$ approaches the difference quotient limit, i.e. the derivative value. However, comparing the behavior of the first and the third mode frequencies (Fig. 12a and b), the convergence of the calculated values is found to worsen for higher frequency states.
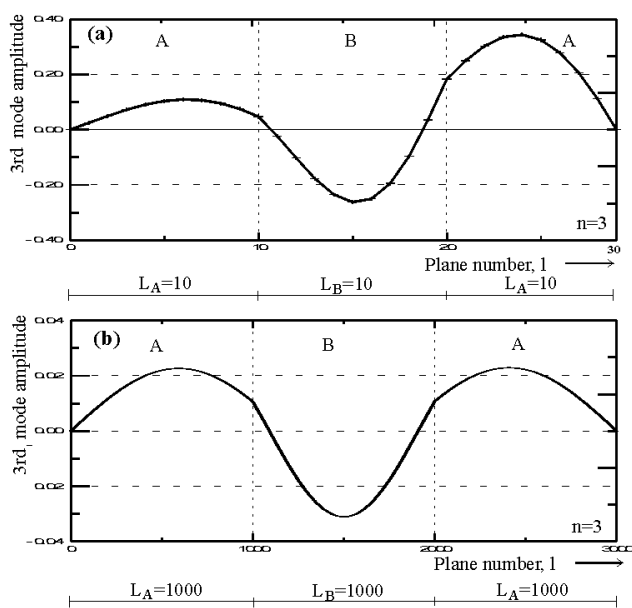

Fig. 13. The 3rd mode normalized profile for a trilayer A/B/A (the same as in Fig. 12) with $\mathrm{A}$ and $\mathrm{B}$ sublayers having equal thicknesses $\left(L_{\mathrm{A}}=L_{\mathrm{B}}\right)$, used as a test for the computation convergence. (a) The solution obtained for $L_{\mathrm{A}}=10$ is not a physical one, since it is not completely symmetric. (b) For $L_{\mathrm{A}}=1000$, a physical (completely symmetric) solution is obtained.

In our work, we were forced to introduce an additional test for convergence of results, based on symmetry properties of the calculated eigenstates. For a symmetrical (multilayer) system (e.g. A/B/A), with symmetrical boundary conditions on the external surfaces, symmetrical and antisymmetrical modes are the only possible eigenstates. However, it turned out that if the number $L_{\mathrm{A}}$ of planes was too small (i.e. a was big), asymmetrical spin-wave mode profiles were obtained (Fig. 13a), though they should be symmetrical; but their symmetry is found to improve as the accuracy improves ( $a$ decreases) (Fig. 13b). The higher the considered mode, the better accuracy is needed to obtain fully symmetrical (or fully antisymmetrical) mode. In our calculations, we found that satisfactory convergence was provided by dividing the unit cell AB into 1000 planes, which, for the unit cell width of $100 \AA$ assumed in this study, corresponds to $a=0.1 \AA$. For higher mode profiles, the accuracy of calculations must be additionally enhanced. 


\section{Acknowledgments}

The present work was supported by the grant No. 2 PO3B 07916 from the State Committee for Scientific Research (Poland) and in part by the French-Polish cooperation program POLONIUM.

\section{References}

[1] J.D. Joannopoulos, R.D. Meade, J.N. Winn, Photonic Crystals, Princeton University Press, New York 1995.

[2] Photonic Band Gap Materials, Ed. C.M. Soukoulis, Kluwer Academic, Dordrecht 1996.

[3] R. Hillebrand, W. Hergert, W. Harms, Phys. Status Solidi B 217, 981 (2000).

[4] A.R. McGurn, Phys. Rev. B 61, 13235 (2000).

[5] M.S. Kushwaha, P. Halevi, G. Martinez, L. Dobrzynski, B. Djafari-Rouhani, Phys. Rev. B 49, 2313 (1994).

[6] J.O. Vasseur, B. Djafari-Rouhani, L. Dobrzynski, M.S. Kushwaha, P. Halevi, J. Phys., Condens. Matter 6, 8759 (1994).

[7] J.O. Vasseur, B. Djafari-Rouhani, L. Dobrzynski, P.A. Deymier, J. Phys., Condens. Matter 9, 7327 (1997).

[8] Y. Tanaka, S. Tamura, Phys. Rev. B 58, 7958 (1998).

[9] Y. Tanaka, S. Tamura, Phys. Rev. B 60, 13294 (1999).

[10] J.O. Vasseur, L. Dobrzynski, B. Djafari-Rouhani, H. Puszkarski, Phys. Rev. B 54, 1043 (1996).

[11] M. Krawczyk, H. Puszkarski, Acta Phys. Pol. A 93, 805 (1998).

[12] M. Krawczyk, H. Puszkarski, Acta Phys. Superficierum 3, 89 (1999).

[13] R.E. Camley, R.L. Stamps, J. Phys., Condens. Matter 5, 3727 (1993).

[14] B. Li, J. Yang, J.L. Shen, G.Z. Yang, J. Phys., Condens. Matter 7, 1405 (1995).

[15] B. Hillebrands, J.V. Harzer, C. Guntherodt, C.D. England, C.M. Falco, Phys. Rev. $B$ 42, 6839 (1990).

[16] B. Hillebrands, Phys. Rev. B 37, 9885 (1988).

[17] R.L. Stamps, R.E. Camley, Phys. Rev. B 54, 15200 (1996).

[18] D.H. Anselmo, M.G. Cottam, E.L. Albuquerque, J. Phys., Condens. Matter 12, 1041 (2000).

[19] M. Jouanne, W. Szuszkiewicz, J.F. Morhange, M.A. Kanehisa, J.M. Hartmann, H. Mariette, E. Dynowska, G. Karczewski, T. Wojtowicz, J. Kossut, J. Barnas, J. Cryst. Growth 184/185, 947 (1998).

[20] H. Puszkarski, Prog. Surf. Sci. 9, 191 (1979).

[21] H. Puszkarski, J.-C.S. Lévy, J. Phys., Condens. Matter 2, 4913 (1990).

[22] H. Puszkarski, Surf. Sci. Rep. 20, 45 (1994).

[23] J.-C.S. Lévy, Surf. Sci. Rep. 1, 39 (1981).

[24] R.W. Damon, J.R. Eshbach, J. Phys. Chem. Solids 19, 308 (1961). 
[25] T. Wolfram, R.E. De Wames, Phys. Rev. B 1, 4358 (1970).

[26] T. Wolfram, R.E. De Wames, Prog. Surf. Sci. 2, 233 (1972).

[27] B.A. Kalinikos, A.N. Slavin, J. Phys. C 19, 7013 (1986).

[28] B.A. Kalinikos, in: Linear and Nonlinear Spin Waves in Magnetic Films and Superlattices, Ed. M.G. Cottam, World Scientific, Singapore 1994, p. 89.

[29] G.T. Rado, R.J. Hicken, J. Appl. Phys. 63, 3885 (1988).

[30] S. Li, L.E. Cross, Phys. Status Solidi B 159, 861 (1990).

[31] B. Hillebrands, Phys. Rev. B 41, 530 (1990). 